\title{
In-Depth Cannabis Multiclass Metabolite Profiling Using Sorptive Extraction and Multidimensional Gas Chromatography with Low- and High-Resolution Mass Spectrometry
}

\author{
Flavio A. Franchina ${ }^{1 *}$, Lena M. Dubois ${ }^{1}$, and Jean-François Focant ${ }^{1}$ \\ ${ }^{1}$ University of Liège, Molecular Systems, Organic \& Biological Analytical Chemistry Group, 11 Allée du Six Août, \\ 4000 Liège, Belgium. \\ *flaviofranchina@gmail.com, ffranchina@uliege.be
}

\section{Table of content}

Supplementary Table S1: Chemical class, compound name, CAS number (CAS\#) and experimental retention index ( $\left.\mathrm{RI}_{\exp }\right)$ of the 27 chemical standards used for chemical class elution classification in the GCxGC system and method validation. Supplementary Table S2: Relative standard deviation (RSD\%) of the chemical classes of interest in the quality control sample under the final SBSE conditions. For the characteristics ions used for measuring the response, refer to Table 2 in the main text.

Supplementary Table S3: Qualitative information (MS library similarity (MS lib. match) and experimental retention index $\left.\left(\mathrm{RI}_{\text {exp }}\right)\right)$ of the compounds reported in Figure 5. For the retention index search, the tolerance was set to \pm 20 units. When a reference retention index was not available and high MS similarity (>800) was obtained, the MS library hit is reported.

Supplementary Table S4: Repeatability and inlet conversion yields (5 replicates) of the acid CBD-A and THC-A to the neutral forms CBD and THC.

Supplementary Table S5: Quantitative information and chemotyping of the sample analyzed. Quantitative values were obtained from 3 replicates and are expressed in $w / w \%$.

Supplementary Figure S1: The 2D plot expansion of the cannabinoids elution zone (sample 7) highlighting the selectivity of GCXGC. I) Separation between chromatographic artifacts (i.e. column bleed) and analytes of interest. II) Resolution of analyte pairs (arrows) which would co-elute in conventional 1D GC.

Supplementary Figure S2: Comparison of flow-modulated GC×GC (lower) and unmodulated GC (upper) traces showing the difference in response magnitude (sesquiterpenes elution zone of sample 8).

Supplementary Figure S3: Workflow of data treatment for the cannabis inflorescences analysis.

Supplementary Figure S4: PC scores plot of the selected 70 features from the cannabis inflorescences. Features were selected amongst the most statistically significant ones (Kruskal-Wallis, $p>0.05$ ), and more expressed at least in one of the sample type. Ellipses represent the $95 \%$ confidence interval for each group.

Supplementary Figure S5A-D: High-resolution mass spectra ( $\geq 25,000 \mathrm{fwhm}$ ) of chlorothalonil (A), cyprodinil (B), bisphenol $G(C)$, and HU-331 (D). 
Supplementary Table S1. Chemical class, compound name, CAS number (CAS\#) and experimental retention index $\left(\mathrm{Rl}_{\text {exp }}\right)$ of the 27 chemical standards used for chemical class elution classification in the $\mathrm{GC} \times \mathrm{GC}$ system and method validation.

\begin{tabular}{|c|c|c|c|}
\hline Chemical class & Name & CAS\# & $\mathbf{R \mathbf { I } _ { \text { exp } }}$ \\
\hline & Cannabidiol (CBD) & $13956-29-1$ & 2452 \\
\hline & Cannabidiolic Acid (CBD-A)* & $1244-58-2$ & - \\
\hline \multirow[t]{10}{*}{ Cannabinoids } & $\Delta 9$-tetrahydrocannabinol (THC) & $972-08-3$ & 2552 \\
\hline & $\Delta 9$-tetrahydrocannabinolic acid A (THC-A)* & $23978-85-0$ & - \\
\hline & Cannabinol (CBN) & $521-35-7$ & 2623 \\
\hline & $\alpha$-pinene & $80-56-8$ & 933 \\
\hline & Camphene & $79-92-5$ & 950 \\
\hline & $\beta$-pinene & $18172-67-3$ & 976 \\
\hline & $\beta$-myrcene & $123-35-3$ & 984 \\
\hline & $\delta$-3-carene & $13466-78-9$ & 1009 \\
\hline & $\alpha$-terpinene & $99-86-5$ & 1017 \\
\hline & $p$-cymene & $99-87-6$ & 1028 \\
\hline \multirow{11}{*}{ Monoterpenes } & Limonene & $5989-27-5$ & 1026 \\
\hline & Eucalyptol & $470-82-6$ & 1033 \\
\hline & Ocimene & $13877-91-3$ & 1049 \\
\hline & $\gamma$-terpinene & $99-85-4$ & 1060 \\
\hline & Terpinolene & $586-62-9$ & 1088 \\
\hline & Linalool & $78-70-6$ & 1099 \\
\hline & Isopulegol & $89-79-2$ & 1146 \\
\hline & Geraniol & $106-24-1$ & 1255 \\
\hline & $\beta$-caryophyllene & $87-44-5$ & 1423 \\
\hline & $\alpha$-humulene & $6753-98-6$ & 1461 \\
\hline & Nerolidol (cis- isomer) & $3790-78-1$ & 1544 \\
\hline \multirow[t]{4}{*}{ Sesquiterpenes } & Nerolidol (trans- isomer) & $35944-21-9$ & 1564 \\
\hline & Caryophyllene oxide & $1139-30-6$ & 1593 \\
\hline & Guaiol & $489-86-1$ & 1608 \\
\hline & $\alpha$-bisabolol & $23089-26-1$ & 1695 \\
\hline
\end{tabular}


Supplementary Information

Supplementary Table S2. Relative standard deviation (RSD\%) of the chemical classes of interest in the quality control sample under the final SBSE conditions. For the characteristics ions used for measuring the response, refer to Table 2 in the main text.

\begin{tabular}{lc} 
Classes & RSD\% $(\mathbf{n}=\mathbf{8})$ \\
\hline Monoterpenes & 18.0 \\
Sesquiterpenes & 7.0 \\
IS (d f $_{5}$-chlorobenzene) & 11.6 \\
Hydrocarbons & 13.2 \\
Terpenoid alcohols and fatty acids & 11.8 \\
Cannabinoids & 18.3 \\
\hline
\end{tabular}


Supplementary Table S3. Qualitative information (MS library similarity (MS lib. match) and experimental retention index $\left(\mathrm{Rl}_{\text {exp }}\right)$ ) of the compounds reported in Figure 5 . For the retention index search, the tolerance was set to \pm 20 units. When a reference retention index was not available and high MS similarity (>800) was obtained, the MS library hit is reported.

\begin{tabular}{|c|c|c|c|}
\hline Label & Tentative ID & $\begin{array}{l}\text { MS lib. } \\
\text { match* }\end{array}$ & $\mathbf{R} \mathbf{I}_{\exp }^{\#}$ \\
\hline Mono.1 & $\alpha$-thujene & 877 & 930 \\
\hline Mono.2* & $\alpha$-pinene* & 898 & 938 \\
\hline Mono.3 & Sabinene & 790 & 987 \\
\hline Mono.4* & $\beta$-myrcene* & 882 & 989 \\
\hline Mono.5 & $\alpha$-phellandrene & 816 & 1012 \\
\hline Mono.6* & Eucalyptol* & 878 & 1037 \\
\hline Mono.7 & $\alpha$-ocimene & 891 & 1055 \\
\hline Mono.8* & $\gamma$-terpinene* & 880 & 1068 \\
\hline Mono.9 & Fenchone & 840 & 1098 \\
\hline Mono.10 & Trans-limonene oxide & 740 & 1131 \\
\hline Mono.11 & Monoterpene - unidentified & 798 & 1152 \\
\hline Mono.12 & Monoterpene - unidentified & 706 & 1158 \\
\hline Mono.13 & Terpinen-4-ol & 865 & 1187 \\
\hline Mono.14 & p-cymen-8-ol & 792 & 1192 \\
\hline Mono.15 & Fenchyl acetate & 754 & 1231 \\
\hline Mono.16 & Monoterpene - unidentified & - & 1321 \\
\hline Sesqui.1 & $\alpha$-copaene & 761 & 1373 \\
\hline Sesqui. 2 & Ylangene & 881 & 1385 \\
\hline Sesqui.3 & $\gamma$-elemene & 710 & 1444 \\
\hline Sesqui.4 & Alloaromadendrene & 829 & 1467 \\
\hline Sesqui.5 & Sesquiterpene - unidentified & - & 1471 \\
\hline Sesqui. 6 & Sesquiterpene - unidentified & - & 1473 \\
\hline Sesqui. 7 & Sesquiterpene - unidentified & - & 1540 \\
\hline Sesqui. 8 & $\alpha$-calacorene & 884 & 1563 \\
\hline Sesqui.9* & Nerolidol* & 901 & 1569 \\
\hline Sesqui.10 & Sesquiterpene - unidentified & - & 1576 \\
\hline Sesqui.11* & Caryophyllene oxide* & 894 & 1599 \\
\hline Sesqui.12 & Sesquiterpene - unidentified & 822 & 1681 \\
\hline Sesqui.13 & Sesquiterpene - unidentified & - & 1689 \\
\hline Sesqui.14 & Sesquiterpene - unidentified & - & 1737 \\
\hline Terp.alc.-fat.ac.1 & Fat.ac. ester - unidentified & - & 1795 \\
\hline Terp.alc.-fat.ac. 2 & Benzoic acid, 2-methylbutyl ester & 800 & 1796 \\
\hline Terp.alc.-fat.ac.3 & Fat.ac. ester - unidentified & - & 1826 \\
\hline Terp.alc.-fat.ac. 4 & Ester (benzoic acid, hept-3-yl ester) ${ }^{\circ}$ & 803 & 1832 \\
\hline Terp.alc.-fat.ac. 5 & Ketone (ketone, methyl 2,2,3-trimethylcyclopentyl) ${ }^{\circ}$ & 801 & 1895 \\
\hline Terp.alc.-fat.ac.6 & Neophytadiene & 839 & 1886 \\
\hline Terp.alc.-fat.ac.7 & Unknown & - & 1903 \\
\hline Terp.alc.-fat.ac. 8 & Fat.ac. ester - unidentified & - & 1991 \\
\hline Terp.alc.-fat.ac. 9 & Fat.ac. ester - unidentified & - & 1998 \\
\hline Terp.alc.-fat.ac.10 & Fat.ac. ester - unidentified & - & 2094 \\
\hline Terp.alc.-fat.ac.11 & Phytol & 893 & 2095 \\
\hline
\end{tabular}


Terp.alc.-fat.ac.12 9-octadecenoic acid, methyl ester

9112108

Terp.alc.-fat.ac.13 Fat.ac. ester - unidentified

2185

Terp.alc.-fat.ac.14

Isoamyl laurate 838

2251

Terp.alc.-fat.ac.15 Fat.ac. ester (stearic acid, 2-hydroxy-1-methylpropyl ester) ${ }^{\circ}$

$802 \quad 2312$

Terp.alc.-fat.ac.16 Fat.ac. ester (9,12,15-octadecatrienoic acid, methyl ester) ${ }^{\circ} \quad 853 \quad 2440$

Terp.alc.-fat.ac.17 Fat.ac. ester (octadecanoic acid, 3-hydroxy-, methyl ester) ${ }^{\circ} \quad 814 \quad 2490$

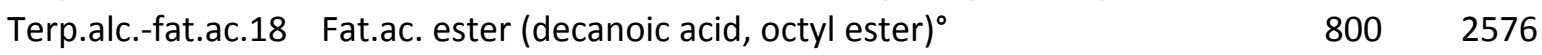

Hydrocarbon.1 Hydrocarbon (decane, 6-ethyl-2-methyl-) ${ }^{\circ} \quad 834 \quad 1146$

Hydrocarbon.2 Hydrocarbon (hexane, 3-ethyl-2,5-dimethyl-) $)^{\circ} \quad 853 \quad 1157$

$\begin{array}{llll}\text { Cannabinoid.1 } & \text { Cannabidivarol }^{\circ} & 842 & 2245\end{array}$

Cannabinoid.2 $\quad$ Cannabinoid (cannabichromene) $^{\circ} \quad 904 \quad 2487$

Cannabinoid.3 $\quad$ Cannabinoid (cannabichromene) ${ }^{\circ} \quad 897 \quad 2669$

Cannabinoid.4 Cannabinoid (11-hydroxy- $\Delta$ 9-tetrahydrocannabinol) ${ }^{\circ} \quad 708 \quad 2666$

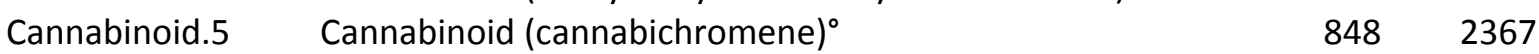

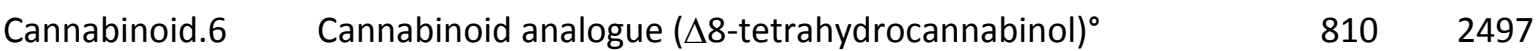

Cannabinoid.7 Cannabinoid analogue ( $\Delta$ 9- tetrahydrocannabinol $)^{\circ} \quad \begin{array}{lll}810 & 2556\end{array}$

$\begin{array}{llll}\text { Unclassified.1 Alcohol (2,4,4-Trimethyl-1-pentanol })^{\circ} & 807 & 1358\end{array}$

Unclassified.2 Ester (2-furanmethanol, tetrahydro-, acetate) ${ }^{\circ} \quad 805 \quad 1605$

Unclassified.3 Unknown $\quad$ - 1812

$\begin{array}{llll}\text { Unclassified.4 Hexadecanal } & 899 & 1823\end{array}$

$\begin{array}{lll}\text { Unclassified.5 Unknown } & - & 1847\end{array}$

$\begin{array}{lll}\text { Unclassified.6 Unknown } & - & 1761\end{array}$

Unclassified.7 Unknown $\quad$ - 2409

$\begin{array}{lll}\text { Unclassified.8 Unknown } & - & 2429\end{array}$

Unclassified.9 Unknown $\quad$ - 2584

$\begin{array}{llll}\text { Unclassified.10 } & \text { Ester (phenethyl tetradecanoate) }^{\circ} & 919 & 2693\end{array}$

$\begin{array}{lll}\text { Unclassified.11 2-phenylethy linoleate } & 831 & 2878\end{array}$

Unclassified.12 Unknown $\quad$ - 2951

Unclassified.13 Unknown $\quad$ - 3132

" Confirmed with analytical-grade standards
- Library hit (>800 MS match) for compounds where the reference retention index was not available 
Supplementary Table S4. Repeatability and inlet conversion yields ( 5 replicates) of the acid CBD-A and THC-A to the neutral forms CBD and THC.

\begin{tabular}{|c|c|c|}
\hline & $\begin{array}{l}\text { Repeatability* } \\
\text { (RSD\%) }\end{array}$ & $\begin{array}{l}\text { Conversion } \\
\text { yield }\end{array}$ \\
\hline CBD & 5.8 & - \\
\hline CBD-A & 15.3 & $61 \% \#$ \\
\hline THC & 4.3 & - \\
\hline THC-A & 10.2 & $56 \%^{\circ}$ \\
\hline
\end{tabular}

Supplementary Table S5. Quantitative information and chemotyping of the analyzed samples. Quantitative values were obtained from 3 replicates and are expressed in $w / w \%$.

\begin{tabular}{|c|c|c|c|c|c|}
\hline & Total CBD & Total THC & CBN & Chemotype* & Terpene dominance $^{\#}$ \\
\hline Sample 1 & $1.80 \pm 0.077$ & $0.08 \pm 0.008$ & $0.03 \pm 0.007$ & CBD-dominant & $\beta$-myrcene/ $\beta$-caryophyllene \\
\hline Sample 2 & $1.92 \pm 0.120$ & $0.07 \pm 0.010$ & $0.02 \pm 0.003$ & CBD-dominant & $\beta$-caryophyllene \\
\hline Sample 3 & $1.50 \pm 0.053$ & $0.06 \pm 0.002$ & $<0.02$ & CBD-dominant & $\beta$-caryophyllene \\
\hline Sample 4 & $1.91 \pm 0.042$ & $0.07 \pm 0.002$ & $0.02 \pm 0.002$ & CBD-dominant & $\beta$-myrcene/Limonene/ $\beta$-caryophyllene \\
\hline Sample 5 & $2.07 \pm 0.130$ & $0.08 \pm 0.003$ & $0.02 \pm 0.001$ & CBD-dominant & $\beta$-myrcene/Limonene/ $\beta$-caryophyllene \\
\hline Sample 6 & $1.75 \pm 0.150$ & $0.07 \pm 0.006$ & $0.02 \pm 0.005$ & CBD-dominant & $\beta$-myrcene/Limonene/ $\beta$-caryophyllene \\
\hline Sample 7 & $5.53 \pm 0.190$ & $0.12 \pm 0.009$ & $0.07 \pm 0.012$ & CBD-dominant & Limonene/ $\beta$-caryophyllene \\
\hline Sample 8 & $7.94 \pm 0.076$ & $0.18 \pm 0.007$ & $0.01 \pm 0.003$ & CBD-dominant & Limonene/ $\beta$-caryophyllene \\
\hline
\end{tabular}




\section{Supplementary Information}

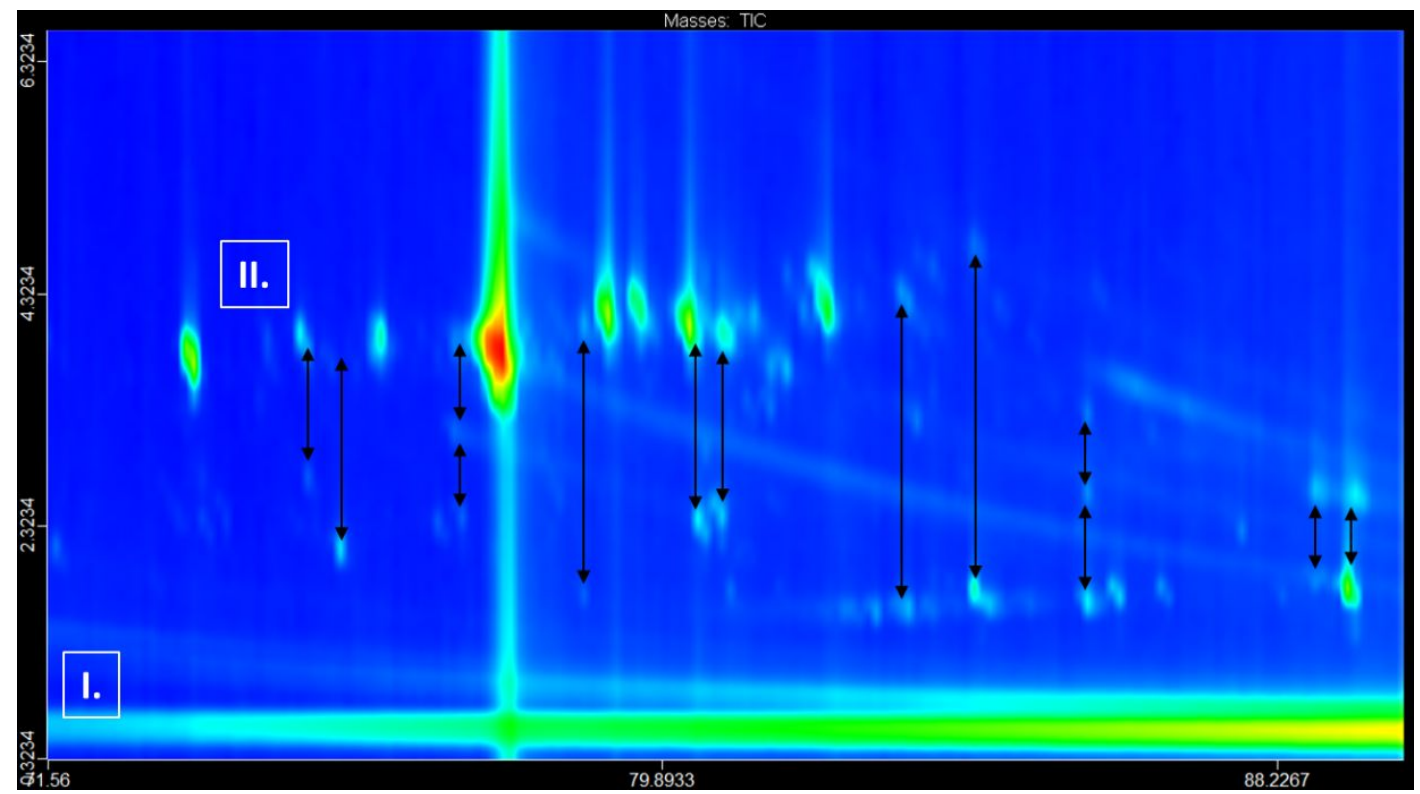

Supplementary Figure S1: The 2D chromatogram expansion of the cannabinoids elution zone (sample 7) highlighting the selectivity of $\mathrm{GC} \times \mathrm{GC}$. I) Separation between chromatographic artifacts (i.e., column bleed) and analytes of interest. II) Resolution of analyte pairs (arrows) which would co-elute in conventional 1D GC.

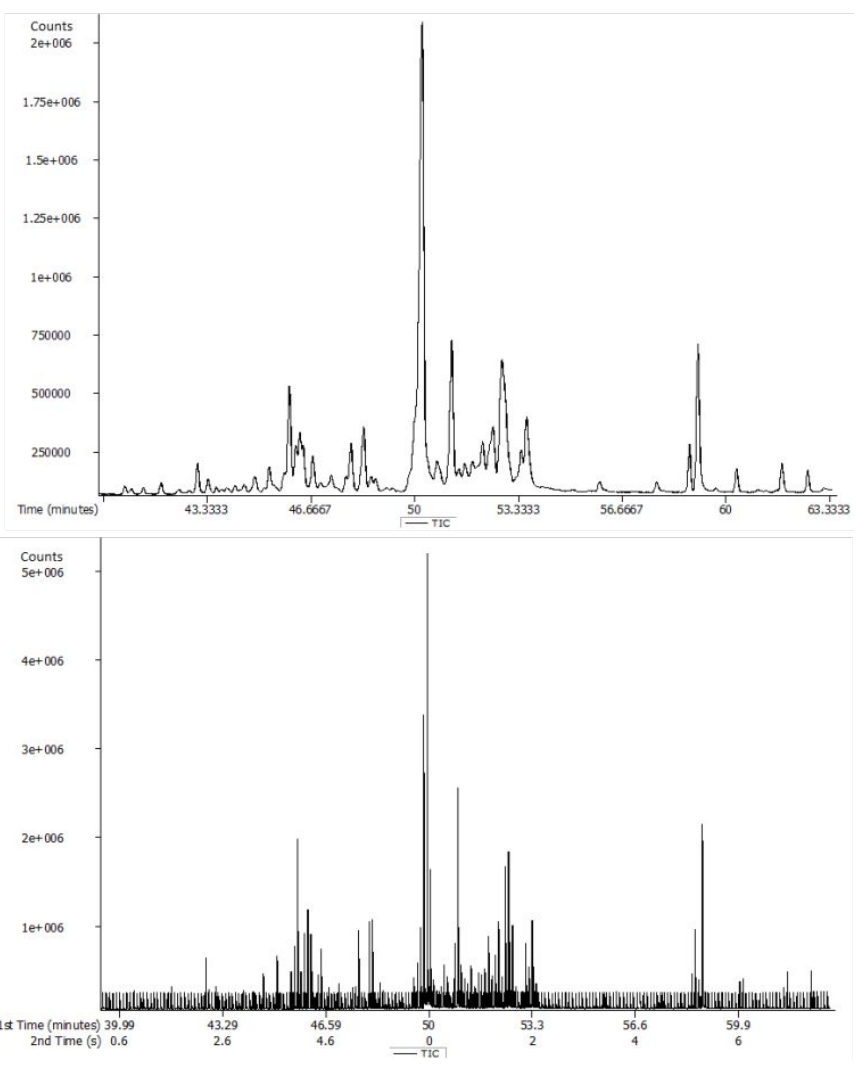

Supplementary Figure S2: Comparison of flow-modulated $\mathrm{GC} \times \mathrm{GC}$ (lower) and unmodulated GC (upper) traces showing the difference in response magnitude (sesquiterpenes elution zone of sample 8). 


\section{Supplementary Information}
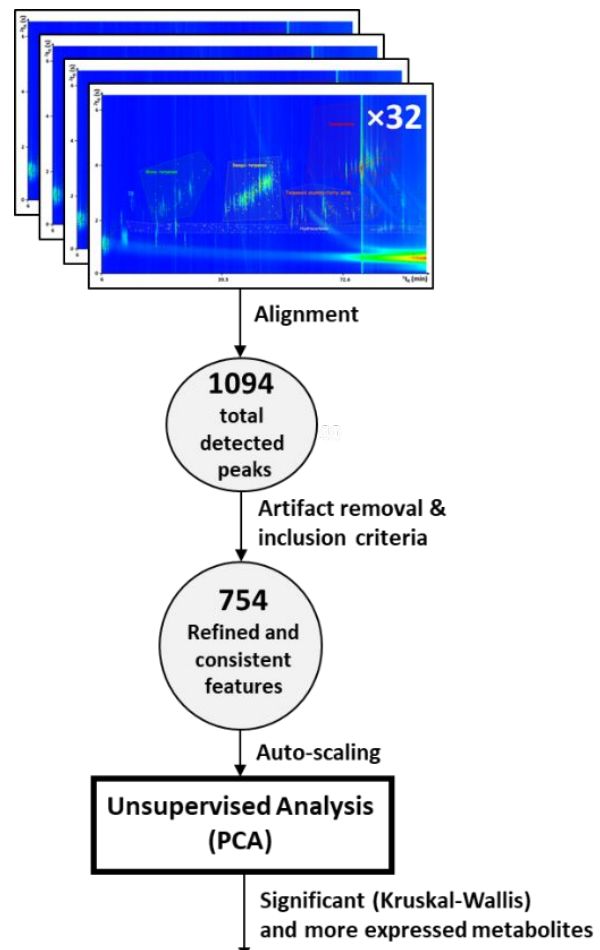

$$
\begin{aligned}
& \text { Supervised Analysis I } \\
& \text { (HCA, PCA) }
\end{aligned}
$$

Supplementary Figure S3: Workflow of data treatment for the cannabis inflorescences analysis.

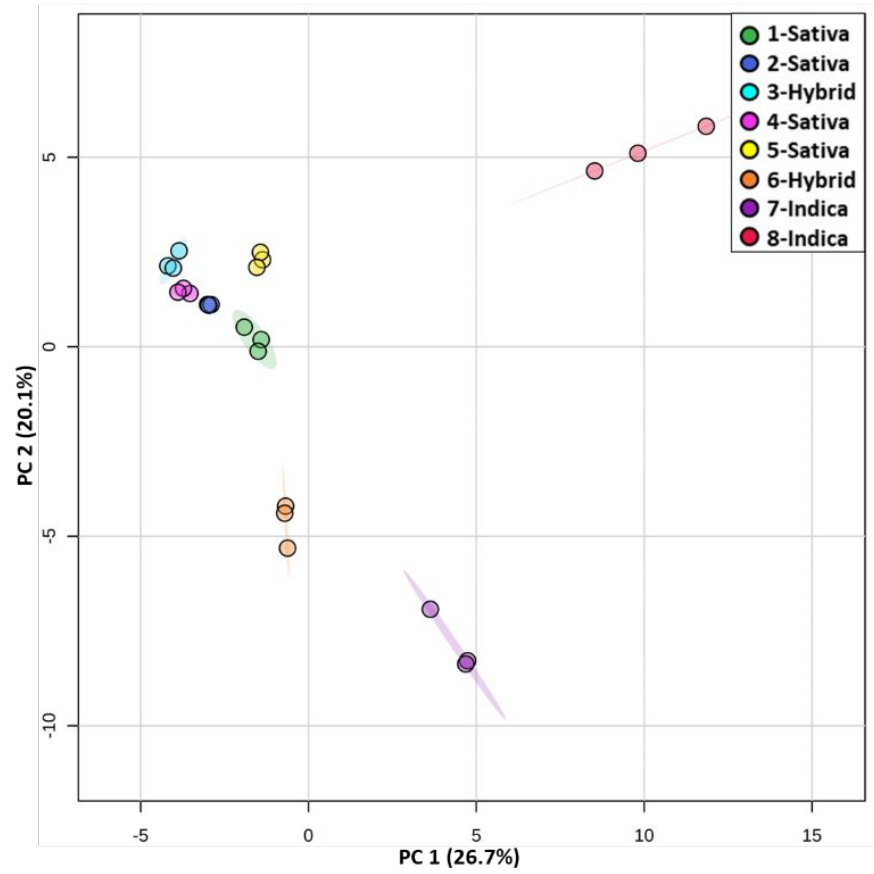

Supplementary Figure S4: PC scores plot of the selected 70 features from the cannabis inflorescences. Features were selected amongst the most statistically significant ones (Kruskal-Wallis, $p>0.05$ ), and more expressed at least in one of the sample type. Ellipses represent the $95 \%$ confidence interval for each group. 

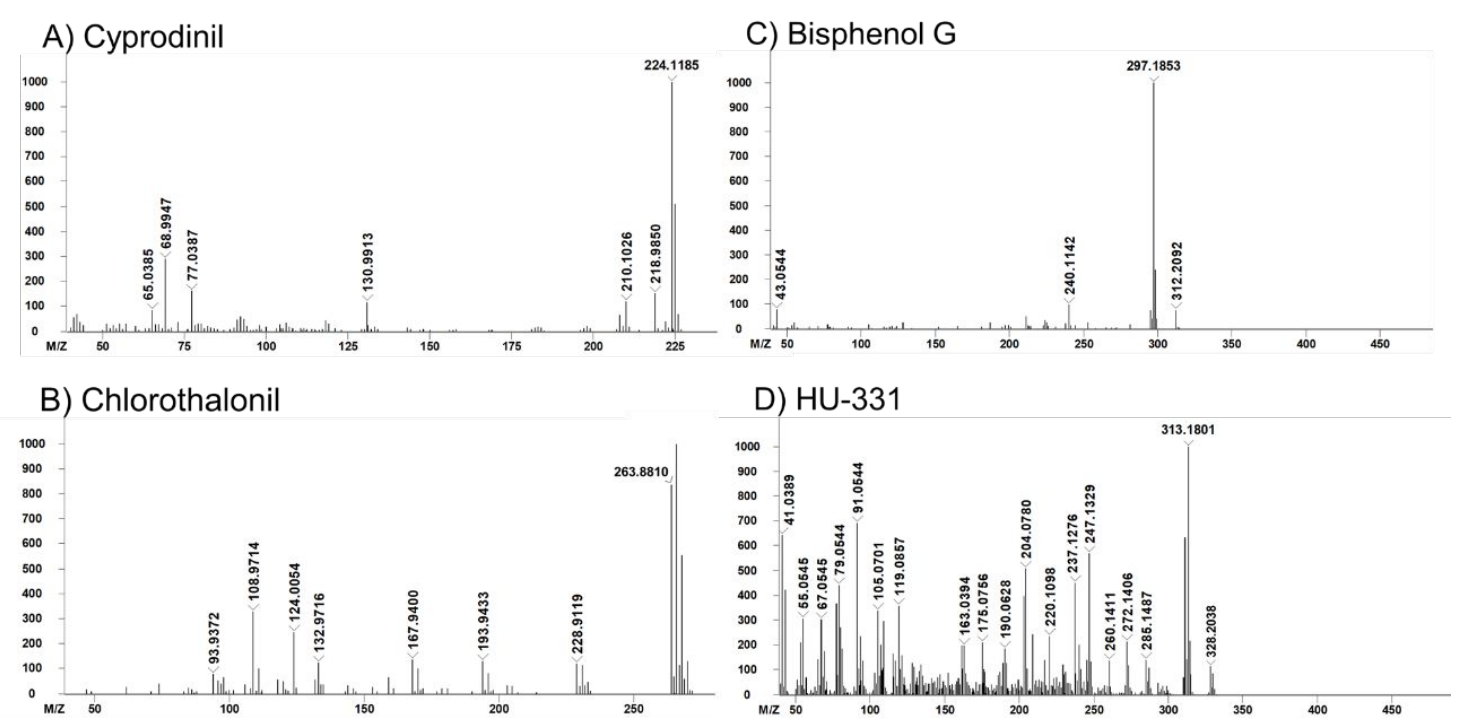

Supplementary Figure S5A-D: High-resolution mass spectra ( $\geq 25,000 \mathrm{fwhm})$ of chlorothalonil (A), cyprodinil (B), bisphenol G (C), and HU-331 (D). 